\title{
CTLA-4-directed Probody BMS-986249
}

National Cancer Institute

\section{Source}

National Cancer Institute. CTLA-4-directed Probody BMS-986249. NCI Thesaurus. Code C146765.

A probody composed of ipilimumab, a recombinant human immunoglobulin (Ig) G1 monoclonal antibody directed against the human T-cell receptor cytotoxic Tlymphocyte-associated antigen 4 (CTLA4; CT LA-4), linked to a proprietary masking peptide that covers the active antigen-binding site of the antibody through a proteasecleavable linker, with potential immune checkpoint inhibitory and antineoplastic activities. Upon administration of CT LA-4-directed probody BMS-986249, the masking peptide is cleaved by tumor-associated proteases upon extravasation into the tumor microenvironment (TME). Protease-mediated removal of the linker enables binding of the unmasked monoclonal antibody moiety to CT LA-4, which is expressed on certain Tcells. This inhibits the CT LA4-mediated downregulation of T-cell activation, and leads to both activation of tumor infiltrating $\mathrm{T}$-effector cells and a cytotoxic T-lymphocyte (CTL)mediated immune response against cancer cells. CT LA4, an inhibitory receptor and member of the immunoglobulin superfamily expressed on activated effector T-cells (T effs) and regulatory T-cells (Tregs), plays a key role in the inhibition of T-cell activity and downregulation of the immune system. The peptide masking of BMS-986249 minimizes binding to CT LA-4 in normal tissues and may reduce systemic toxicity, when compared to ipilimumab. Tumor-associated proteases are present in high concentrations and aberrantly activated in the TME. 